OPEN ACCESS

Edited by:

Zhongheng Zhang,

Zhejiang University, China

Reviewed by:

Hong-Qiu Gu,

Capital Medical University, China

LuKe,

Naniing General Hospital of Nanjing

Military Command, China

Jianfeng Xie,

Southeast University, China

*Correspondence:

Xin-juan Wu

wuxinjuan@sina.com

Jing Sun

j.sun@griffith.edu.au

${ }^{\dagger}$ These authors have contributed equally to this work

Specialty section

This article was submitted to Infectious Diseases - Surveillance,

Prevention and Treatment,

a section of the journal

Frontiers in Public Health

Received: 28 May 2019

Accepted: 25 July 2019

Published: 13 August 2019

Citation:

Jiao J, Yang $X, L i Z$, Zhao $Y$, Cao J, Li F, Liu Y, Liu G, Song B, Jin J, Liu Y, Wen $X$, Cheng S, Yang L, Wu X and Sun $J(2019)$ Incidence and Related

Factors for Hospital-Acquired Pneumonia Among Older Bedridden

Patients in China: A Hospital-Based Multicenter Registry Data Based

Study. Front. Public Health 7:221

doi: 10.3389/fpubh.2019.00221

\section{Incidence and Related Factors for Hospital-Acquired Pneumonia Among Older Bedridden Patients in China: A Hospital-Based Multicenter Registry Data Based Study}

\author{
Jing Jiao ${ }^{1 t}$, Xiang-yun Yang ${ }^{2 t}$, Zhen $\mathrm{Li}^{1}$, Yan-wei Zhao ${ }^{1}$, Jing Cao ${ }^{1}$, Fang-fang $\mathrm{Li}^{1}$, \\ Ying Liu ${ }^{1}$, Ge Liu ${ }^{1}$, Bao-yun Song ${ }^{3}$, Jing-fen Jin ${ }^{4}$, Yi-lan Liu ${ }^{5}$, Xian-xiu Wen ${ }^{6}$, \\ Shou-zhen Cheng ${ }^{7}$, Lin-lin Yang ${ }^{8}$, Xin-juan $\mathrm{Wu}^{1 *}$ and Jing Sun ${ }^{2,9 *}$ \\ ${ }^{1}$ Chinese Academy of Medical Sciences, Peking Union Medical College, Peking Union Medical College Hospital, Beijing, \\ China, ${ }^{2}$ Beijing Key Laboratory of Mental Disorders, The National Clinical Research Center for Mental Disorders, Beijing \\ Anding Hospital, Capital Medical University, Beijing, China, ${ }^{3}$ Henan Provincial People's Hospital, Zhengzhou, China, ${ }^{4}$ The \\ Second Affiliated Hospital, Zhejiang University School of Medicine, Hangzhou, China, ${ }^{5}$ Wuhan Union Hospital, Wuhan, \\ China, ${ }^{6}$ Sichuan Provincial People's Hospital, Chengdu, China, ${ }^{7}$ The First Affiliated Hospital, Sun Yat-sen University, \\ Guangzhou, China, ${ }^{8}$ School of Nursing, Qingdao University, Qingdao, China, ${ }^{9}$ School of Medicine, Griffith University, \\ Gold Coast, QLD, Australia
}

Objective: To identify the incidence and related factors for hospital-acquired pneumonia (HAP) among older bedridden patients in China.

Study design and setting: This multicenter registry data-based study conducted between November 2015 and March 2016 surveyed 7,324 older bedridden patients from 25 hospitals in China (six tertiary, 12 non-tertiary, and seven community hospitals). The occurrence of HAP among all participants was monitored by trained investigators. Demographics, hospitalization information and comorbidity differences were compared between patients with and without HAP. A multilevel regression analysis was used to explore the factors associated with HAP.

Results: Among 7,324 older bedridden patients, 566 patients were diagnosed with HAP. The incidence of HAP in this study was 13.9 per 1,000 person-days. There were statistical differences in gender, age, length of bedridden days, BMI, smoking, department, undergoing general anesthesia surgery, ventilator application, Charlson comorbity index (CCl) score, disturbance of consciousness, tranquilizer use, glucocorticosteroid use, and antibiotic use between patients with HAP and patients without HAP (all $p<0.05$ ). Multilevel regression analysis found no significant variance for HAP at the hospital level $(0.332, t=1.875, p>0.05)$. There were significant differences for the occurrence of HAP among different departments $(0.553, t=4.320$, $p<0.01)$. The incidence density of HAP was highest in the ICU $(30.1 \%$ ) among the selected departments, followed by the departments of neurosurgery $(18.7 \%$ ) and neurology medicine (16.6\%). Individual patient-level factors, including older age, disturbance of consciousness, total $\mathrm{CCl}$ score, ICU admission, and glucocorticoid and antibiotic use, were found to be associated with the occurrence of HAP (all $p<0.05$ ). 


\begin{abstract}
Conclusion: A relatively high incidence density of HAP among older bedridden patients was identified, as well as several factors associated with HAP among the population. This suggests that attention should be paid to the effective management of these related factors of older bedridden patients to reduce the occurrence of HAP.
\end{abstract}

Keywords: hospital-acquired pneumonia, older bedridden patients, incidence density, related factors, registry data-based study

\section{INTRODUCTION}

The number of bedridden patients increases with the increasing prevalence and incidence of diseases among older population (1). Staying in bed at hospital could cause many complications, and pneumonia is one of the most common complications. Hospital-acquired Pneumonia (HAP) is a major nosocomial infection worldwide resulting in increased morbidity, mortality, and medical costs. Older bedridden patients, whose basic physiological needs are carried out in bed, often accompanied by worse immune, swallowing and respiratory function, are at high risk of HAP (2). Approximately $1.1-1.5 \%$ of all hospital patients developed HAP (3), and 5.8-8.3\% of older hospitalized patients developed HAP in western countries $(2,4)$. In China, the incidence of hospital- acquired infections in all hospitalized patients was $3.22-5.22 \%$, of which hospital acquired lower respiratory tract infections were $1.76-11.94 \%$. Patients over 65 years old are the main group of HAP, accounting for about $70 \%$ (5). The incidence of and related factors of HAP among older bedridden patients is still not clear in China.

Identifying the factors associated with HAP is important to prevent HAP and reduce the incidence of HAP. This may ultimately decrease length of hospital stay, mortality, reduce inappropriate antibiotic use, and to improve functional outcomes. Several studies have identified age, smoking, chronic pulmonary disease, type of surgery, malnutrition, state of unconsciousness during hospitalization, mechanical ventilation, use of a nasogastric tube, multi-trauma and poor health condition are potential risk factors for the HAP (6-8). These studies mainly investigated the risk factors for ventilator- associated pneumonia in intensive care units (ICUs). Several studies have investigated the incidence of HAP in non-ICU patients. They found the incidence of HAP outside the ICU was 24 cases per 1,000 patients. The risk factors associated with HAP in non-ICU patients include age, malnutrition, steroid use, chronic renal failure, anemia, unconscious, comorbidity, recent hospitalization, and thoracic surgery (9-11). These findings suggest that the risk factors for HAP vary among patients in different wards. Therefore, it is important to understand the occurrence of and factors associated with HAP in various departments for the prevention of nosocomial infections.

In hospitals, nurses are responsible for most of the life care, nursing and treatment of bedridden patients. Identifying the risk factors of HAP is important for improving the quality of nursing and therapeutic effect of bedridden patients. However, our previous study surveyed the nurses' knowledge and attitudes on complications of bedridden patients including HAP, and found their knowledge on the related factors are not adequate
(12). Therefore, it is necessary to understand the incidence of HAP and related factors among bedridden patients in China. To our best knowledge, no study has estimated the incidence of HAP among older hospitalized bedridden patients in general wards. It is not clear which factors contribute to bedridden HAP in China. To fill this research gap, this study aimed to identify the prevalence and related factors of HAP among older bedridden patients in various departments (ICU, orthopedics, neurology medicine, neurosurgery, general surgery, cardiovascular, other surgery and other medicine) in China by using a nationwide multicenter hospital-based study sample during November 2015 to March 2016.

\section{METHODS}

\section{Study Design, Setting, and Population}

This multicenter study was designed to assess the incidence of HAP in participants during hospitalization. The data used in the current study is a part of a national research program that aimed to construct a standardized nursing intervention model for major complications of immobility (MCI) among bedridden patients. This study adopted a multi-level and purposive sampling method which is a convenient in nature but has clear inclusion criteria to achieve the purpose of the representation of the sample. All departments were embedded within hospitals, and hospitals were within provinces. To meet the inclusion criteria at all level's data, first, six provinces or municipality cities were selected including Beijing, Henan province, Hubei province, Zhejiang province, Guangdong province and Sichuan province to represent the North, South, West, East and Central parts of China. Second, one of the largest tertiary hospital with over 2,000 beds in each province and at least two non-tertiary hospitals in each province, with total 25 hospitals that met the inclusion criteria, were recruited. Third, the department in which older bedridden patients accounted for more than $20 \%$ of the total number of patients was selected from each hospital, including ICU, neurology medicine and other medicine, neurosurgery, general surgery and other surgery, orthopedics and cardiovascular departments. From November 1, 2015 to March 17, 2016, participants who met the inclusion criteria were continuously recruited into the study (specific sampling methods see Figure 1). Bedridden Patients were defined as all patients whose basic physiological needs were carried out in bed because of their illness or weakness except active or passive bedside standing/wheelchair use when examination or treatment was conducted. Patients were recruited if they (1) were 65 years old and over; (2) had stayed in bed for at least $24 \mathrm{~h}$ when he/she was 


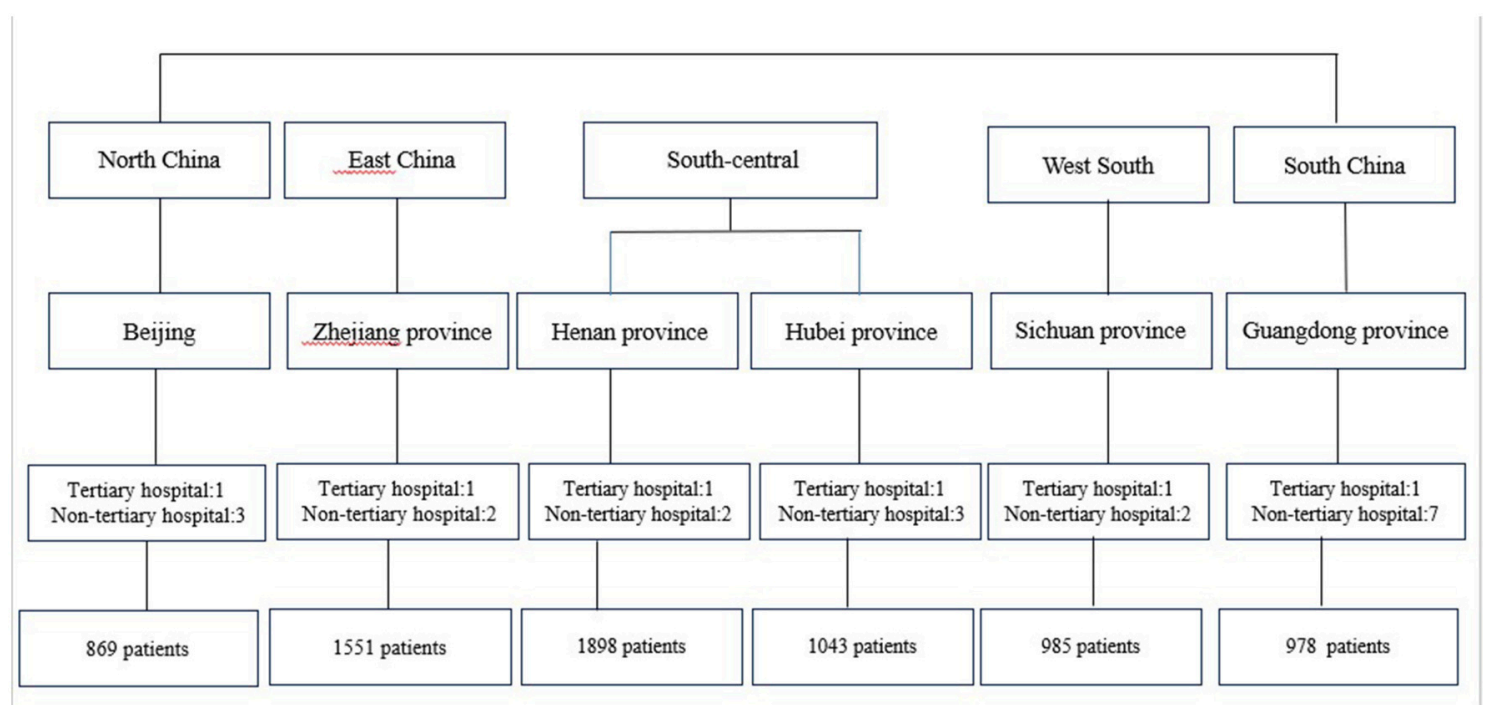

FIGURE 1 | Sampling recruitment procedure.

surveyed; (3) understood the aims of the study and signed the consent form. Patients were excluded from data analysis if they had community-acquired pneumonia and more than one type of MCI at the time of enrollment.

\section{Variables and Data Collection}

HAP refers to pulmonary parenchymal infection caused by pathogenic bacteria existing in hospital environment, which occurs $48 \mathrm{~h}$ after the patient's admission. Ventilator- associated pneumonia (VAP) was included in this study based on the practice guideline for HAP/VAP in China. HAP was diagnosed based on a combination of clinical, laboratory and radiological data by physicians and were recorded on the medical records (5). Patients' socio-demographic characteristics were collected using a questionnaire. To ensure detailed information for age, education and BMI was analyzed, age was categorized into five groups, namely a 65-69 years old group, a 70-74 years old group, a 75-80 years old group, a $80-85$ years old group, and a $\geq 85$ years old group. The education level of patients was divided into illiteracy, primary school, middle school, senior high school, college and above. The grouping of age and education level was based on the distribution of the participants, which ensures that each group has enough sample size to increase the balance between groups. BMI was then used to divide them into 18.5$23.9,<18.5,>23.9$ and $<28$, and $<28 \mathrm{~kg} / \mathrm{m}^{2}$ groups according to the guidelines for prevention and control of overweight and obesity in Chinese adults (13). The smoking status of patients was classified as never smoked, smoking and ex-smokers. Medical comorbidities assessment and hospitalization information use were collected from the audits of the hospital chart and electronic health records. The Charlson comorbidity index (CCI) score was calculated as an important measure of baseline comorbid conditions of the patients. All patients' treatments during hospitalization, including general anesthesia surgery, ventilator use, tranquilizer use, antibiotic use, and glucocorticoid use, were investigated using yes or no questions. The number of bedridden days, hospitalization time, and days from admission to diagnosis of HAP, and other related medical information of all patients, were extracted from medical records and recorded. A case report form and an Electronic Data Collection system (EDC) were designed for the data collection work for this study.

The trained nurses collected data of all participants from admission to discharge. To guarantee the quality of the data collection for the study, a project manual was developed. Two registered nurses in each department were trained by the project team members were responsible for bedridden patient data collection, including recording the patient bedridden status and diagnosis of pneumonia on a daily basis. The head nurses of each department were responsible for information checking and review. In addition, a project team regularly conduct on-site quality control and case verification and provide feedback via email, phone, and meeting.

\section{Ethical Considerations}

This study was approved by the Ethical Committee of Peking Union Medical College Hospital (S-700). Patients received verbal and written information about the study and provided written consent to participate. If patients had a cognitive impairment (e.g., because of dementia), their contact person was asked to provide the consent. Patients were advised they have right to withdraw from the study at any time, and the care they received would not be affected by their participation status. All data were kept confidential and processed anonymously.

\section{Data Analysis}

First, we explored data distribution. Continuous variables were examined for the normality. Then the normal data was presented 
as mean and standard deviation (SD), non-normal data was presented as median and interquartile range (IQR). Dichotomous variables were described as frequency and percentage (14). Second, in the univariate analysis, demographics, hospitalization information, and comorbidity differences between patients with and without HAP groups were assessed using the Student's $t$-test for continuous variables and the Chi-squire test for categorical variables. Considering the lengths of stay in hospital may affect the occurrence of HAP, we calculated the incidence density of HAP using the total number of person-days as denominator. All statistical tests were performed at an alpha level of 0.05 . Two-tailed estimates of effect and 95\% confidence interval were reported for all regression coefficients. SPSS version 24 was used to analyze the data.

Further, we analyzed the association between HAP and the variables determined by clinical significance and significant results of univariate analysis (including age; gender; BMI; smoking status; undergoing general anesthesia surgery; ventilator application; ICU experience; CCI total score; use of antibiotics, tranquilizers, and glucocorticosteroids; and other related factors). A multilevel model approach was considered to explore factors that were associated with the incidence of HAP, because of the nested structure of the data (i.e., patients within different departments and departments within hospitals). Thus, a multilevel regression analysis was performed to determine factors associated with the occurrence of HAP by using MLwin software, version 2.10 (15). The occurrence of HAP was treated as the dependent variable. A random effects model was used to analyze the association between risk factors and HAP at three levels simultaneously so that the contribution of individual-, department-, and hospital -level data to the occurrence of HAP was partitioned.

\section{RESULTS}

\section{Characteristics of All Patients and Incidence of HAP}

A total of 7,324 patients were contacted. All of them had complete data and were included in this analysis. Baseline characteristics of all patients were shown in Table 1. Of all patients, males accounted for $50.9 \%$. The mean age of all patients was 74.51 years (SD $=7.35$ ), and $32.5 \%$ of patients had primary school qualification. Regarding the department, $21.93 \%$ of patients came from ICU, $28.06 \%$ from orthopedics, $19.18 \%$ from neurology, $7.62 \%$ from neurosurgery, $7.05 \%$ from general surgery, and $6.23 \%$ from cardiovascular. The average length of hospital stay was 16.39 days $(\mathrm{SD}=16.18) ; 1,386$ patients spent 15 days or more in hospital, of which 285 patients suffered from HAP, accounting for $50.35 \%$ of all HAP patients. The percentage of patients with a BMI of $18.5-23.9$ was $50.44 \%$. In addition, $78.22 \%$ of patients had no smoking experience and $36.78 \%$ underwent general anesthesia surgery. A total of 478 patients received ventilator support and 770 patients had a CCI score of $>7$. Most patients $(86.85 \%)$ were free from disorders of consciousness; in addition, $13.79 \%$ used glucocorticosteroids, $63.49 \%$ used antibiotics, and $13.90 \%$ took tranquilizers.
Among all participating patients, 566 patients were diagnosed with HAP after they were admitted to hospital. The incidence density of HAP of all participants was 13.9 per 1,000 person-days and it varied among the different departments. The incidence density of HAP in the ICU was 30.1 per 1,000 person-days, which was the highest. Neurosurgery (18.7\%o) and neurology medicine (16.6\%o) also had higher incidence density than other departments (see Table 3). As shown in Figure 2, the median of duration from admission to diagnosis of HAP was 7 days (IQR, 4-13) and the mode was 2 days. We compared the demographic characteristics and clinical data between patients with HAP and patients without HAP, and found that there were statistical differences in gender, age, length of bedridden days, BMI, smoking, department, undergoing general anesthesia surgery, ventilator application, CCI score, disorders of consciousness, tranquilizer use, glucocorticosteroid use, and antibiotic use (all $p<0.05)$ (Table 1).

\section{Factors Associated With HAP}

The above factors with inter-group differences were subsequently entered into the multilevel regression model. Because different nursing conditions may affect the occurrence of HAP, the bed to -nurse ratio (attained or not attained) was considered in the model. The multilevel regression model included three levels: individual, department, and hospital. As shown in Table 2, there was no significant variance for HAP at the hospital level (0.332, $t=1.875, p>0.05)$. There was significant variance for HAP among different departments $(0.553, t=4.320, p<$ 0.01). The patients from the ICU had higher risk for HAP than those from non-ICU departments (1.182, $t=6.031, p$ $<0.001)$. Patients over 75 years of age were at increased risk compared with those $<70$ years $(p<0.05)$. Patients with disorders of consciousness were at higher risk compared with those without disorders of consciousness (1.146, $t=$ $8.815, p<0.001)$. High CCI score, use of antibiotics, and use of glucocorticoids were related to the occurrence of HAP (all $p<0.05$ ), while patients who received general anesthesia for surgical intervention had lower risk than those who did not $(p<0.01)$. There was no association between smoking, gender, BMI, length of bedridden days, bed-to-nurse ratio, ventilator application, and tranquilizer use and the occurrence of HAP (all $p>0.05$ ).

These individual level factors contributed to the occurrence of HAP and explained $81.98 \%$ of variance.

\section{Distribution of Factors Associated With HAP Among Different Departments}

In the multilevel analysis, the hospital contributed to $6.13 \%$ of the variance, but its contribution to the occurrence of HAP was not significant. The department explained $11.88 \%$ of the variance and significantly contributed to the occurrence of HAP (see Table 2). Because of the significant variance of HAP explained by the department-level results, we further analyzed the distribution of factors associated with HAP in patients with HAP among different departments. As shown in Table 3, there were significant differences in the distribution 
TABLE 1 | Baseline characteristics of older patients with hospital acquired pneumonia and without hospital acquired pneumonia $(n=7,324)$.

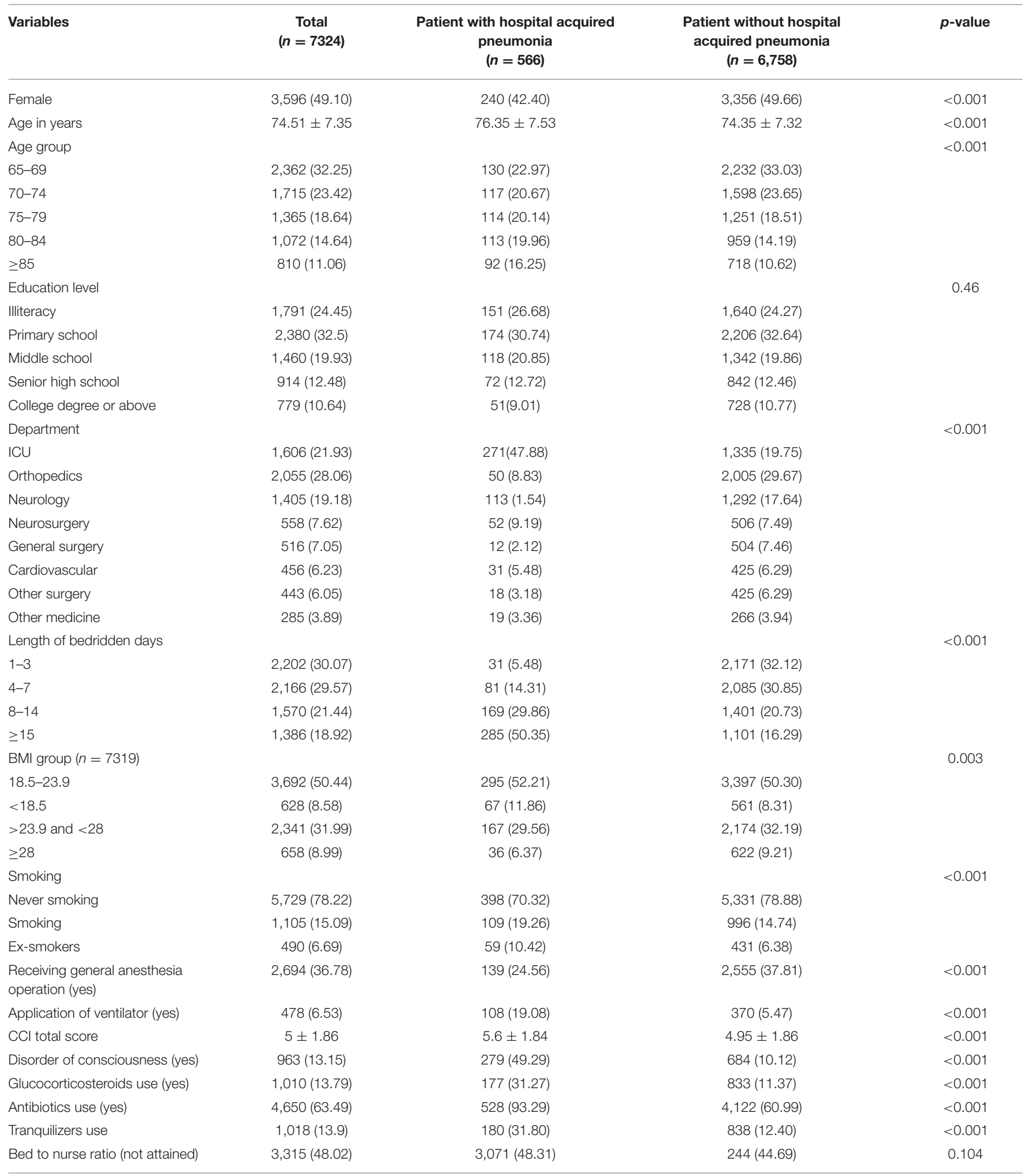

CCI, Charlson Comorbidity Index; ICU, Intensive care unit.

of age, disorders of consciousness, CCI total score, undergoing general anesthesia surgery, use of glucocorticoids, and use of antibiotics in patients with HAP among different departments (all $p<0.001$ ). The results showed that $59.5 \%$ of disorders of consciousness were in HAP patients in the ICU department, $18.3 \%$ in HAP patients in the neurology medicine department, 


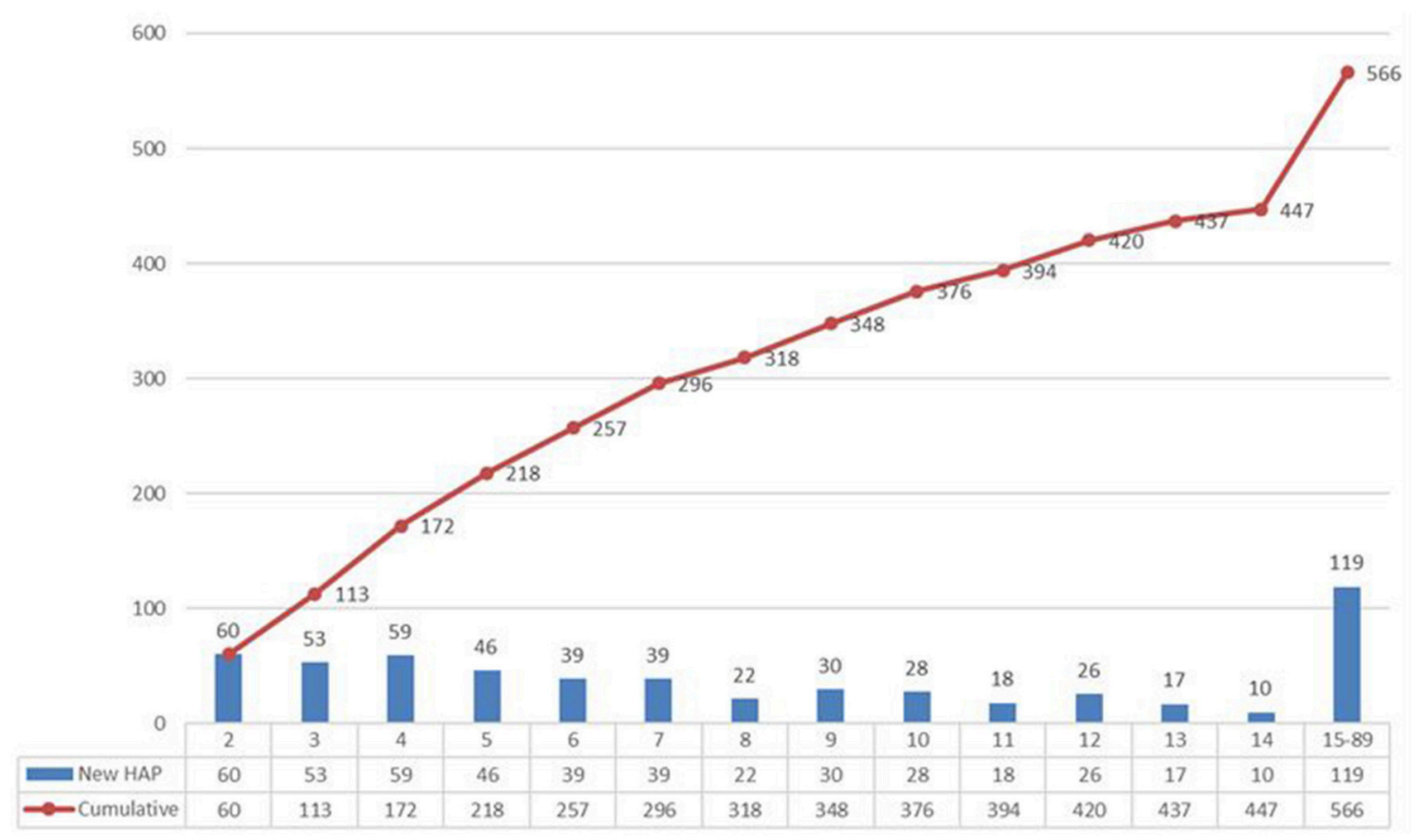

FIGURE 2 | Daily and cumulative of occurrence of HAP in patients with HAP. The median of duration from admission to diagnosis of HAP was 7 days (IQR, 4-13) and the mode was 2 days.

and $14.0 \%$ in HAP patients in the neurosurgery department. The use of glucocorticoids was higher in the ICU (54.2\%) and neurosurgery (14.1\%) departments compared with other departments. The use of antibiotics was relatively high in the ICU (49.8\%), neurology medicine (18.0\%), neurosurgery (9.1\%), and orthopedics $(8.5 \%)$ departments. Most patients with HAP who received general anesthesia for surgical intervention came from the ICU $(50.4 \%) ; 16.5 \%$ came from the orthopedics department and $15.8 \%$ from the neurosurgery department. Higher CCI total score was found in the ICU $(5.7 \pm 2.1)$, neurology medicine $(5.3 \pm 1.3)$, general surgery $(5.8 \pm 2.7)$, cardiovascular $(5.5 \pm 1.5)$, other surgery (5.4 \pm 2.4$)$, and other medicine $(5.1 \pm 1.7)$ departments. Older age was found in the orthopedics, neurology medicine, general surgery, other medicine, and ICU departments.

\section{DISCUSSION}

HAP is one of the leading hospital-acquired infections worldwide and is associated with elevated morbidity, mortality and increased hospital costs (1). Few studies have been conducted on the epidemiology of HAP among older bedridden patients when the incidence of HAP in the older people increases with age (16). This study aimed to identify the incidence and related factors of HAP among older bedridden patients by using representative departments in 25 hospitals with different administrative levels in six provinces and cites in China. Our study demonstrates that the incidence density of HAP among older bedridden patients is as high as $13.9 \%$ person-days; this is consistent with the results from other countries that the incidence of HAP is between 8 and $10 \%$ of patients admitted to hospital for the older people units $(16,17)$. There was no significant variance for HAP at the hospital level in this study. Thus, the current result is the first time to demonstrate the incidence of HAP among older bedridden patients in China. The incidence of HAP varied among different departments. In line with previous studies, we found that the incidence rate of HAP was highest in the ICU among the selected departments in this study (7), followed by the departments of neurosurgery and neurology.

Our study found that age, disturbance of consciousness, total CCI score, ICU admission, glucocorticoid use, and antibiotic use were associated with occurrence of bedridden related HAP. Previous studies have also shown that older age and unconsciousness are associated with $\operatorname{HAP}(9,18,19)$. Older bedridden patients often have complicated illnesses and have to accept multiple types of medicines, such as glucocorticoids, and antibiotics. It is possible that long-term use of glucocorticoids reduces the body's immune function and inhibits neutrophil chemotaxis $(1,20)$. In this study, most patients $(437 / 566)$ had antibiotic use before diagnosing HAP. This is consistent with other studies showing that antibiotic use is a common risk factor for $\operatorname{HAP}(1,21)$. The improper use of antibiotics has resulted in the imbalance of bacterial flora and the emergence of multi-drug-resistant strains, especially in hospitals, which has increased the incidence of nosocomial infections (22). Proper use of antibiotics can help to decrease the infection rate of HAP. 
TABLE 2 | Multilevel regression analysis of factors associated with HAP.

\begin{tabular}{|c|c|c|c|c|c|c|}
\hline Variables & Estimation & Standard error & $t$ ratio & $P$-value & $\begin{array}{l}\text { Hospital level } \\
\text { estimate (SE) }\end{array}$ & $\begin{array}{c}\text { Department level } \\
\text { estimate (SE) }\end{array}$ \\
\hline Constant & -3.867 & 0.334 & -11.578 & $<0.001$ & $0.332(0.177)$ & $0.553(0.128)$ \\
\hline Age & & & & & $T=1.875$ & $T=4.320$ \\
\hline $70-74$ & 0.166 & 0.134 & 1.239 & $>0.05$ & $P>0.05$ & $P<0.01$ \\
\hline $75-79$ & 0.308 & 0.143 & 2.154 & $<0.05$ & & \\
\hline $80-84$ & 0.425 & 0.150 & 2.833 & $<0.05$ & & \\
\hline 85 or more & 0.524 & 0.166 & 3.157 & $<0.05$ & & \\
\hline Male & 0.098 & 0.107 & 0.916 & $>0.05$ & & \\
\hline Length of bedridden days & -0.000 & 0.000 & 0 & $>0.05$ & & \\
\hline \multicolumn{7}{|l|}{ BMI } \\
\hline$<18.5$ & 0.174 & 0.157 & 1.108 & $>0.05$ & & \\
\hline $24-27.9$ & 0.022 & 0.106 & 0.207 & $>0.05$ & & \\
\hline$\geq 28$ & -0.035 & 0.180 & -0.194 & $>0.05$ & & \\
\hline Ex-smokers & 0.069 & 0.137 & 0.504 & $>0.05$ & & \\
\hline Smoking & 0.106 & 0.173 & 0.613 & $>0.05$ & & \\
\hline $\mathrm{ICU}$ & 1.182 & 0.196 & 6.031 & $<0.001$ & & \\
\hline Receiving general anesthesia operation & -0.767 & 0.128 & -5.992 & $<0.01$ & & \\
\hline Application of ventilator & 0.259 & 0.159 & 1.629 & $>0.05$ & & \\
\hline Disorder of consciousness & 1.146 & 0.130 & 8.815 & $<0.001$ & & \\
\hline Glucocorticosteroids use & 0.619 & 0.122 & 5.073 & $<0.01$ & & \\
\hline Antibiotics use & 2.211 & 0.180 & 12,283 & $<0.001$ & & \\
\hline Tranquilizers use & 0.060 & 0.136 & 0.441 & $>0.05$ & & \\
\hline $\mathrm{CCl}$ total score & 0.057 & 0.026 & 2.192 & $<0.05$ & & \\
\hline Bed to nurse ratio (attained) & 0.152 & 0.159 & 0.956 & $>0.05$ & & \\
\hline Total variance explained & $\begin{array}{c}\text { Individual level } \\
81.98 \%\end{array}$ & & & & $6.13 \%$ & $11.88 \%$ \\
\hline
\end{tabular}

CCI, Charlson Comorbidity Index; ICU, Intensive care unit; BMI, body mass index.

TABLE 3 | The incidence density of HAP among different departments and distribution of related factors of HAP in patients with HAP among different departments.

\begin{tabular}{|c|c|c|c|c|c|c|c|c|c|c|}
\hline Variables & ICU & Orthopedics & $\begin{array}{l}\text { Neurology } \\
\text { medicine }\end{array}$ & Neurosurgery & $\begin{array}{l}\text { General } \\
\text { surgery }\end{array}$ & Cardiovascula & $\begin{array}{l}\text { ar Other } \\
\text { surgery }\end{array}$ & $\begin{array}{c}\text { Other } \\
\text { medicine }\end{array}$ & $\chi^{2} / F$ & $p$ \\
\hline Disorder of consciousness & $166(59.4 \%)$ & $9(3.2 \%)$ & $51(18.3 \%)$ & $39(14.0 \%)$ & $3(1.1 \%)$ & $6(2.2 \%)$ & $3(1.1 \%)$ & $2(0.7 \%)$ & 82.67 & $<0.001$ \\
\hline Glucocorticosteroids use & $96(54.2 \%)$ & 17 (9.6\%) & $14(7.9 \%)$ & $25(14.1 \%)$ & $3(1.7 \%)$ & $5(2.8 \%)$ & $9(5.1 \%)$ & $8(4.6 \%)$ & 35.43 & $<0.001$ \\
\hline Antibiotics use & 263 (49.8\%) & $45(8.5 \%)$ & 95 (18.0\%) & $48(9.1 \%)$ & $12(2.3 \%)$ & $28(5.3 \%)$ & $18(3.4 \%)$ & 19 (3.6\%) & 26.35 & $<0.001$ \\
\hline $\mathrm{CCl}$ total score & $5.7 \pm 2.1$ & $4.1 \pm 1.2$ & $5.3 \pm 1.3$ & $4.3 \pm 1.3$ & $5.8 \pm 2.7$ & $5.5 \pm 1.5$ & $5.4 \pm 2.4$ & $5.1 \pm 1.7$ & 164.41 & $<0.001$ \\
\hline Age in years & $75.6 \pm 7.5$ & $79.9 \pm 7.5$ & $77.5 \pm 6.9$ & $73.9 \pm 6.8$ & $79.9 \pm 7.1$ & $69.5 \pm 4.2$ & $76.8 \pm 8.7$ & $76.3 \pm 7.5$ & 6.98 & $<0.001$ \\
\hline $\begin{array}{l}\text { Incidence density Mean } \\
\text { (SD) }\end{array}$ & $30.1 \%$ (0.087) & $4.0 \%$ (0.034) & $16.6 \%$ (0.069) & 18.7\%о (0.073) & $2.1 \%$ (0.018) & $\begin{array}{l}10.8 \% \\
(0.050)\end{array}$ & 3.3\%o (0.018) & $\begin{array}{l}13.1 \% \\
(0.058)\end{array}$ & \multicolumn{2}{|c|}{$\begin{array}{c}\text { Total incidence } \\
\text { density } \\
13.9 \%\end{array}$} \\
\hline
\end{tabular}

CCI, Charlson Comorbidity Index; ICU, Intensive care unit.

There has been new progress in the use of antibiotics. New evidence-based guidelines for the diagnosis and treatment of these entities were released by the Infectious Diseases Society of America and the American Thoracic Society in 2016, which sets 7 days as the recommended duration of antibiotic therapy, regardless of the isolated pathogen(s). The available data suggest there was no difference between short-course (7-8 days) and long-course (10-15 days) antibiotic regimens in regard to mortality (8). The reduction of the antibiotic use will reduce days of exposure to antibiotics, minimizing a risk of developing resistance in bedridden patients (23). These findings suggest that antibiotics and glucocorticoids should be used cautiously during the treatment.

The Charlson comorbidity index, a method of predicting mortality by classifying or weighting comorbid conditions (comorbidities), has been widely utilized to measure disease burden, severity and prognosis since it was published in 1987 (24). Comorbidities were considered as an important predictor of mortality of pneumonia in older patients as the more comorbidities the patients have, the more susceptible they are to have infection (25-28). Our study also found higher CCI total score was associated with the occurrence of HAP. 
Thus, a comprehensive assessment of complications should be made at the beginning of hospitalization for older patients to prevent HAP.

It is noteworthy that we found that patients receiving general anesthesia for surgical intervention had a decreased risk of HAP. This finding may be explained by the fact that general anesthesia requires patients to have good cardiopulmonary function and be able to withstand general anesthesia. Therefore, the physical condition of these patients may be better than that of patients who cannot undergo general anesthesia, and they are less likely to suffer pneumonia infection because of their suitability for more comprehensive treatment and care, such as sputum aspiration after completion of general anesthesia surgery (29). The reasonable and effective treatment of primary diseases and rapid recovery from the disease may be the key factors to prevent nosocomial infection. Further research is needed to demonstrate the association between general anesthesia surgery and the occurrence of HAP.

In this study, $47.88 \%$ of patients with HAP had ICU experience, and the multilevel regression analysis indicated that the ICU department was significantly associated with the occurrence of HAP. It was reported that HAP is the most common infection acquired in the ICU (9). Previous research showed that patients who had been in the ICU for 3-4 days were three times more likely to be infected than those with a stay of 1-2 days, and those who had been in the ICU for 21 days or more were at 33 times the risk of patients with a hospital stay of 1-2 days (30). ICU patients usually have more serious illness and accept more invasive operations, such as nasal feeding, ventilation, and these operations may increase infection risk to patients (31). This study also found that patients in the ICU had the most HAP-related factors, including higher age and CCI total score, disturbance of consciousness, and use of glucocorticoids and antibiotics, which might also explain why the incidence of HAP in the ICU was the highest. We also found higher incidence of HAP in the department of neurosurgery and neurology medicine. Most patients in theses departments had severe diseases, such as stroke, epilepsy, brain tumor, brain injury and cerebral hemorrhage, which may also have high CCI total score, disturbance of consciousness, and the use of glucocorticoids and antibiotics. These factors were found to be significantly related to HAP. Current findings suggest we should also focus on the prevention of HAP in the department of neurosurgery and neurology medicine while we prevent the occurrence of HAP in ICU.

The current study found differences in gender, BMI, smoking, length of bedridden days, ventilator application, and use of tranquilizers between patients with HAP and patients without HAP. Several previous studies demonstrated that BMI, smoking, ventilator application, and use of tranquilizers were associated with the occurrence of HAP (32-36). However, our study did not find a correlation between these factors and HAP. The inconsistent results may be due to differences in the sampling method and subjects included in this study. The results of this study can only represent the situation of older bedridden patients in China.

This study investigated the incidence and related factors of HAP among older bedridden patients in China. This study included older bedridden patients from different types of department of 25 hospitals in only six provinces and municipalities, a more representative sample from other provinces is needed to generalize the results to other regions in China in future studies. Furthermore, we excluded patients who had more than one type of MCI at the time of enrollment. Thus, the incidence of HAP may be underestimated.

\section{CONCLUSION}

In conclusion, the incidence of HAP among older bedridden patients is as high as 13.9 per 1,000 person days in this study. The incidence density of HAP varies among different departments. In addition to the ICU, the department of neurosurgery and neurology medicine have relatively high incidence of HAP. Several factors including age, disturbance of consciousness, ICU admission, CCI total score, antibiotic use, and glucocorticoid use are associated with HAP. Current results suggest that effective evaluation and management of these related factors of HAP in older bedridden patients should be intensified to reduce the occurrence of HAP.

\section{DATA AVAILABILITY}

All datasets generated for this study are included in the manuscript/supplementary files.

\section{AUTHOR CONTRIBUTIONS}

XW designed the study. JS designed the statistical methodology, analyzed the data, reviewed, and edited the manuscript. ZL conducted data analysis. JJia and XY wrote the manuscript. YZ, JC, FL, YiL, GL, BS, JJin, Yi-lL, XW, SC, and LY collected the data. All authors critically reviewed and proofed the manuscript.

\section{FUNDING}

This work was supported by the National Health and Family Planning Commission (Beijing, China) [grant number 201502017]. The funders had no role in study design, data collection and analysis, decision to publish, or preparation of the manuscript.

\section{ACKNOWLEDGMENTS}

We thank all the patients who participated in the study and the nurses who carried out the survey. 


\section{REFERENCES}

1. Li C, Duan J, Liu S, Meng X, Fu C, Zeng C, et al. Assessing the risk and disease burden of Clostridium difficile infection among patients with hospital-acquired pneumonia at a University Hospital in Central China. Infection. (2017) 45:621-8. doi: 10.1007/s15010-0171024-1

2. Burton LA, Price R, Barr KE, McAuley SM, Allen JB, Clinton $\mathrm{AM}$, et al. Hospital-acquired pneumonia incidence and diagnosis in older patients. Age Ageing. (2016) 45:171-4. doi: 10.1093/ageing/ afv168

3. Ewan V, Hellyer T, Newton J, Simpson J. New horizons in hospital acquired pneumonia in older people. Age Ageing. (2017) 46:352-8. doi: 10.1093/ageing/afx029

4. Rosa RG, Goldani LZ, dos Santos RP. Risk factors for multidrug-resistant bacteremia in hospitalized cancer patients with febrile neutropenia: a cohort study. Am J Infect Control. (2014) 42:74-6. doi: 10.1016/j.ajic.2013. 06.025

5. Shi Y, Huang Y, Zhang T-T, Cao B, Wang H, Zhuo C, et al. Infection Study Group of Chinese Thoracic Society, Chinese Medical Association. Guidelines for the diagnosis and treatment of hospital-acquired pneumonia and ventilator-associated pneumonia in adults in China (2018 edition). Chin J Tuberc Respir. (2018) 41:255-80.

6. Vincent JL, Lobo S, Struelens M. Ventilator associated pneumonia: risk factors and preventive measures. J Chemother. (2001) 13:211-7. doi: 10.1179/joc.2001.13.Supplement-2.211

7. Guzman-Herrador B, Molina CD, Allam MF, Navajas RF. Independent risk factors associated with hospital-acquired pneumonia in an adult ICU: 4-year prospective cohort study in a university reference hospital. J Public Health (Oxf). (2016) 38:378-83. doi: 10.1093/pubmed/f dv042

8. Kalil AC, Metersky ML, Klompas M, Muscedere J, Sweeney DA, Palmer LB, et al. Management of adults with hospital-acquired and ventilator-associated pneumonia: 2016 clinical practice guidelines by the Infectious Diseases Society of America and the American Thoracic Society. Clin Infect Dis. (2016) 63:e61-111. doi: 10.1093/cid/ ciw353

9. Cakir Edis E, Hatipoglu ON, Yilmam I, Eker A, Tansel O, Sut $\mathrm{N}$. Hospital-acquired pneumonia developed in non-intensive care units. Respiration. (2009) 78:416-22. doi: 10.1159/0002 32392

10. Sopena N, Sabrià M, Neunos 2000 Study Group. Multicenter-study of hospital-acquired pnumonia in non-ICU patients. Chest. (2005) 127:213-9. doi: 10.1378/chest.127.1.213

11. Sopena N, Heras E, Casas I, Bechini J, Guasch I, Pedro-Botet ML, et al. Risk factors for hospital-acquired pneumonia outside the intensive care unit: a case-control study. Am J Infect Control. (2014) 42:38-42. doi: 10.1016/j.ajic.2013.06.021

12. Li Z, Zhou X, Cao J, Li Z, Wan X, Li J, et al. Nurses' knowledge and attitudes regarding major immobility complications among bedridden patients: a prospective multicentre study. J Clin Nurs. (2018) 27:1969-80. doi: 10.1111 /jocn. 14339

13. Obesity CWGo. Guildelines for prevention and control of overweight and obesity in Chinese adults. Acta Nutr Sinica. (2004) 26:1-4. doi: 10.3321/j.issn:0512-7955.2004.01.001

14. Zhang Z. Univariate description and bivariate statistical inference: the first step delving into data. Ann Transl Med. (2016) 4:91. doi: 10.21037/atm.2016.02.11

15. Spencer NH, Jeremy M. Statistical software for microcomputers MLwiN and Mplus. Br J Math Stat Psychol. (2001) 54:383-7. doi: 10.1348/000711001159528

16. Stolbrink M, McGowan L, Saman H, Nguyen T, Knightly R, Sharpe J, et al. The early mobility bundle: a simple enhancement of therapy which may reduce incidence of hospital-acquired pneumonia and length of hospital stay. J Hosp Infect. (2014) 88:34-9. doi: 10.1016/j.jhin.2014. 05.006
17. El Solh AA, Bhora M, Pineda L, Dhillon R. Nosocomial pneumonia in elderly patients following cardiac surgery. Respir Med. (2006) 100:729-36. doi: 10.1016/j.rmed.2005.07.011

18. van der Maarel-Wierink CD, Vanobbergen JN, Bronkhorst EM, Schols JM, de Baat C. Risk factors for aspiration pneumonia in frail older people: a systematic literature review. J Am Med Dir Assoc. (2011) 12:344-54. doi: 10.1016/j.jamda.2010.12.099

19. Bi J, Yang J, Wang Y, Yao C, Mei J, Liu Y, et al. Efficacy and safety of adjunctive corticosteroids therapy for severe community-acquired pneumonia in adults: an updated systematic review and metaanalysis. PLoS ONE. (2016) 11:e0165942. doi: 10.1371/journal.pone.01 65942

20. Zhu H, Zhao Z, Zhou Y, Chen X, Li Y, Liu X, et al. High-dose glucocorticoid aggravates TBI-associated corticosteroid insufficiency by inducing hypothalamic neuronal apoptosis. Brain Res. (2013) 1541:69-80. doi: 10.1016/j.brainres.2013.10.002

21. Sandoval CP. Short-course versus prolonged-course antibiotic therapy for hospital-acquired pneumonia in critically ill adults. Crit Care Nurse. (2016) 36:82-3. doi: $10.4037 / \mathrm{ccn} 2016840$

22. Chung DR, Song JH, Kim SH, Thamlikitkul V, Huang SG, Wang H, et al. High prevalence of multidrug-resistant nonfermenters in hospital-acquired pneumonia in Asia. Am J Respir Crit Care Med. (2011) 184:1409-17. doi: 10.1164/rccm.201102-0349OC

23. Lyons PG, Kollef MH. Prevention of hospital-acquired pneumonia. Curr Opin Crit Care. (2018) 24:370-8. doi: 10.1097/MCC.00000000000 00523

24. Quan H, Li B, Couris CM, Fushimi K, Graham P, Hider P, et al. Updating and validating the Charlson comorbidity index and score for risk adjustment in hospital discharge abstracts using data from 6 countries. Am J Epidemiol. (2011) 173:676-82. doi: 10.1093/aje/ kwq433

25. Ma HM, Tang WH, Woo J. Predictors of in-hospital mortality of older patients admitted for community-acquired pneumonia. Age Ageing. (2011) 40:736-41. doi: 10.1093/ageing/afr087

26. Cabre M, Serra-Prat M, Palomera E, Almirall J, Pallares R, Clave P. Prevalence and prognostic implications of dysphagia in elderly patients with pneumonia. Age Ageing. (2010) 39:39-45. doi: 10.1093/ageing/a fp100

27. Ho $\mathrm{CH}$, Chen YC, Chu CC, Wang JJ, Liao KM. Age-adjusted Charlson comorbidity score is associated with the risk of empyema in patients with COPD. Medicine (Baltimore). (2017) 96:e8040. doi: 10.1097/MD.0000000000008040

28. Frenkel WJ, Jongerius EJ, Mandjes-van Uitert MJ, van Munster BC, de Rooij SE. Validation of the Charlson comorbidity index in acutely hospitalized elderly adults: a prospective cohort study. J Am Geriatr Soc. (2014) 62:342-6. doi: 10.1111 /jgs. 12635

29. Kazaure HS, Martin M, Yoon JK, Wren SM. Long-term results of a postoperative pneumonia prevention program for the inpatient surgical ward. JAMA Surg. (2014) 149:914-8. doi: 10.1001/jamasurg.201 4.1216

30. Vincent J-L, Bihari DJ, Suter PM, Bruining HA, White J, Wolff M, et al. The prevalence of nosocomial infection in intensive care units in Europe results of the European Prevalence of Infection in Intensive Care (EPIC) Study. JAMA. (1995) 274:639-44. doi: 10.1001/jama.1995.035300800 55041

31. Rello J, Oster G, Vera-Llonch M, Bellm L, Redman R, Kollef MH, VAP Outcomes Scientific Advisory Group. Epidemiology and outcomes of ventilator-associated pneumonia in a large US database. Chest. 122:2115-21. doi: 10.1378/chest.122.6.2115

32. Obiora E, Hubbard R, Sanders RD, Myles PR. The impact of benzodiazepines on occurrence of pneumonia and mortality from pneumonia: a nested case-control and survival analysis in a populationbased cohort. Thorax. (2013) 68:163-70. doi: 10.1136/thoraxjnl-2012202374

33. Huemer HP, Lassnig C, Nowotny N, Irschick EU, Kitchen M, Pavlic M. Diazepam leads to enhanced severity of orthopoxvirus infection and 
immune suppression. Vaccine. (2010) 28:6152-8. doi: 10.1016/j.vaccine.2010. 07.032

34. Sanders RD, Godlee A, Fujimori T, Goulding J, Xin G, SalekArdakani S, et al. Benzodiazepine augmented gamma-amino-butyric acid signaling increases mortality from pneumonia in mice. Crit Care Med. (2013) 41:1627-36. doi: 10.1097/CCM.0b013e3182 $7 \mathrm{c} 0 \mathrm{c} 8 \mathrm{~d}$

35. Almirall J, Bolibar I, Serra-Prat M, Roig J, Hospital I, Carandell E, et al. New evidence of risk factors for community-acquired pneumonia: a populationbased study. Eur Respir J. (2008) 31:1274-84. doi: 10.1183/09031936.000 95807

36. Timsit JF, Esaied W, Neuville M, Bouadma L, Mourvllier B. Update on ventilator-associated pneumonia. F1000Res. (2017) 6:2061. doi: 10.12688/f1000research.12222.1
Conflict of Interest Statement: The authors declare that the research was conducted in the absence of any commercial or financial relationships that could be construed as a potential conflict of interest.

The handling editor declared a shared affiliation, though no other collaboration, with one of the authors JJin.

Copyright (c) 2019 Jiao, Yang, Li, Zhao, Cao, Li, Liu, Liu, Song, Jin, Liu, Wen, Cheng, Yang, Wu and Sun. This is an open-access article distributed under the terms of the Creative Commons Attribution License (CC BY). The use, distribution or reproduction in other forums is permitted, provided the original author(s) and the copyright owner(s) are credited and that the original publication in this journal is cited, in accordance with accepted academic practice. No use, distribution or reproduction is permitted which does not comply with these terms. 